\title{
A Validity and Reliability Study of a Chinese Assessment Tool for Persons with Moderate to Severe Intellectual Disabilities
}

\author{
Christopher L.F. Chan ${ }^{1, *}$, Claudia Lai ${ }^{2}$ and Iris $\mathrm{Chi}^{3}$ \\ ${ }^{1}$ New Life Psychiatric Rehabilitation Association, The Wellness Centre, Kowloon, Hong Kong \\ ${ }^{2}$ School of Nursing, Hong Kong Polytechnic University, Hunghom, Hong Kong \\ ${ }^{3}$ School of Social Work, University of Southern California, Los Angeles, CA
}

\begin{abstract}
This study was designed to test the psychometric properties of the Chinese interRAI Intellectual Disability (ID) tool in a Chinese population with learning disabilities in Hong Kong. The Chinese interRAI ID was prepared based on the original interRAI ID which is a standardized, comprehensive instrument and is designed to evaluate the strengths, preferences, and needs of persons with all levels of ID living in various care settings. A sample of 100 people with moderate to severe intellectual disabilities was assessed with the Chinese interRAI ID and its criterion measures. The subscales of the interRAI ID, including the Cognitive Performance Scale, Depression Rating Scale, Aggressive Behavior Scale, Activities of Daily Living Hierarchy Scale, and Instrumental Activities of Daily Living Involvement Scale, had high internal consistency (Cronbach's $\alpha=.66$ to .87 ) and test-retest reliability $(r=.96$ to $.99 ; \mathrm{K}=.68$ to .81 ). Comparison of the interRAI ID scales with criterion measures supported concurrent and discriminant validity of these scales. The study results provide preliminary support for the Chinese interRAI ID as a reliable and valid tool for assessing Chinese individuals with learning disabilities in Hong Kong.
\end{abstract}

Keywords: interRAI Intellectual Disability, validation, reliability, validity, Chinese.

\section{INTRODUCTION}

Clinicians and researchers are increasingly recognizing that people with intellectual disabilities (ID) often experience difficulties in multiple domains. Using standardized assessment instruments to measure status and functioning in various areas helps identify the service needs of people with ID and assists clinicians in intervention planning. Several multidimensional scales have been devised to evaluate the functional skills, behaviors, and abilities of people with ID; for example, the American Association on Mental Retardation Adaptive Behavior Scale and the Vineland Scales of Adaptive Behavior. Both instruments are designed to measure the adaptive behaviors of individuals with ID or developmental disabilities and are used extensively in Western countries [1, 2]. More recently, a new assessment tool, interRAI Intellectual Disability, has been developed. InterRAl is an international collaboration of 50 researchers and clinicians from more than 23 countries who aspire to improve the quality and efficiency of care delivery in health care services by developing a family of clinical assessments. The Resident Assessment Instrument-Minimum Data Set for residential care was one of the original tools developed by interRAl and is now mandatory in nursing homes in North America and other regions. Some interRAI tools have been adopted

*Address correspondence to this author at the Wellness Centre (YTM), 2/F, 332 Nam Cheong Street, Kowloon, Hong Kong; Tel: (852) 3552 5440;

Fax: (852) 3552 5353; E-mail: clfchan@gmail.com worldwide and translated into different languages, such as Dutch, Spanish, Italian, and Estonian.

The interRAI ID is an administered rating scale designed for evaluating the needs, strengths, and preferences of people with intellectual disabilities living in community- and facility-based settings [3]. The standardized minimum assessment captures 13 key domains critical to evaluating a person with ID in a holistic manner; e.g., health and mental health status, maladaptive behaviors, social support, and abilities. The assessment is based on information collected from a variety of sources, including direct observation; discussion with the individual, the individual's family and support network, and staff; and available documentation. The findings of interRAI ID not only illustrate the characteristics of an individual but also support the development of comprehensive care and service planning drawn from systematic reviews of international literature and expertise on best-practice guidelines in various areas. In addition, the instrument includes well-researched scales used to evaluate an individual's current clinical status; for example, the Activities of Daily Living (ADL) Hierarchy Scale, Depression Rating Scale (DRS), and Cognitive Performance Scale (CPS) [4-6]. Furthermore, the interRAI ID also collects information useful for measuring individual outcomes, evaluating the quality of services provided, and determining the resource intensity of service needs. 
The interRAI ID has recently gained popularity in Western countries. Thus, the interRAI ID is a potentially useful addition to existing instruments available for people with ID. The psychometric data available for the use of interRAI ID among people with ID have been generally established [7]. The results of the study showed moderate correlations between interRAI ID's scales and criterion measures. In addition, another study has demonstrated that a newly developed scale embedded in the interRAI ID, the Aggressive Behavior Scale (ABS), has good concurrent validity with other scales measuring similar concepts [8].

In Hong Kong, a validated Chinese standardized instrument that captures the multidimensional domains of people with ID is lacking. In view of the possible clinical utility of the interRAI ID as a routine measure for service providers to document and monitor changes in people with ID in the Hong Kong context, this paper describes the translation of the instrument into the Chinese and the psychometric evaluation of 5 selected scales embedded in the Chinese interRAI ID among people with moderate and severe ID. Specifically, the internal consistency, test-retest reliability, and concurrent and discriminant validity of the 5 interRAI ID scales (ADL Hierarchy Scale, IADL Involvement Scale, CPS, DRS, and ABS) were examined.

\section{METHOD}

\section{Setting}

Study participants were recruited from hostels for people with either moderate or severe mental disabilities. Both types of hostels offered residential service to people with mental disabilities with different levels of support. The former is designed for people with moderate mental disabilities who are capable of basic self-care but are unable to live independently in the community, whereas the latter provides housing for people with severe mental disabilities who lack basic self-care skills and require assistance in personal and nursing care. However, the allocation of people with mental disabilities into these two types of hostels is not solely based on their degree of mental disabilities but also their self-care abilities.

\section{Sampling}

A sample of 100 people with intellectual disabilities was randomly recruited from 3 hostels (operated by a nongovernmental organization in Hong Kong) that offer 153 and 108 placements for people with moderate and severely mental disabilities, respectively. Sample size estimation was based on the following assumptions: significance criterion $=.05$ (one tail), power size $=.9$, and correlation coefficient $=.3$, which referred to the correlation coefficient between DRS and chart diagnoses [9]. According to Cohen [10], the sample size should not be fewer than 92. To account for an estimated $10 \%$ dropout rate, 100 study participants were randomly selected. Potential participants were eligible if: (1) they were diagnosed by a physician to have intellectual disabilities; (2) they were 18 years old or older; and (3) they had resided in a hostel for people with moderate mental disabilities or a hostel for people with severe mental disabilities for at least 6 months. Residents who were mentally incapacitated and whose guardianship order was appointed to the director of Hong Kong's Social Welfare Department were excluded.

\section{Data Collection}

All professional staff involved in caring for the selected participants, including social workers, nurses, occupational therapists, and physiotherapists, were involved in data collection. Staff members were required to receive a 2-day training on the objectives and methodology of the study and ways to administer the interRAI ID and criterion measures. During the training, staff members reviewed each item of the interRAI ID, including its definition, scoring method, and criteria, to ensure their ability to administer the interRAI ID consistently with its manual. Trainings were delivered by the first and second authors and an agency staff member with experience in delivering interRAl instrument training. Following the training session, staff completed an examination that involved rating a hypothetical case using interRAI ID. Those who correctly answered $80 \%$ or more of the exam items were considered to be qualified raters of interRAI ID. After all staff members passed the examination, they administered the interRAI ID and criterion measures to the recruited participants. The same trained raters readministered the interRAI ID 3 weeks later to 30 study participants who were randomly selected from the pool of study participants. Ethical approval was obtained from a local university.

\section{Instruments}

\section{Preparing a Chinese Version of the interRAI ID}

In this study, data collection consisted of two stages: translation of the interRAI ID into Chinese (Hong Kong) and validation of the Chinese interRAI ID. 
In the first stage, the Chinese interRAI ID was prepared basing on the original version and the Taiwan version of interRAI ID. To the best of our knowledge, the Taiwan version of interRAI ID has not been validated. Due to the marked difference in dialects spoken by people living in Hong Kong and Taiwan, the first author modified the Taiwan version with reference to the original version, giving conceptual equivalence priority over word-for word linguistic equivalence. Revision of items including rewording of terms and rearrangement of sentence structures ensured semantic equivalence between the original and Chinese versions and improved comprehensibility for Chinese residents of Hong Kong. No alteration of item content was made except for those items relating to personal information, such as Medicare number, language dialect, and type of residential setting. The early version of the instrument was reviewed by a panel of bilingual health care professionals in the intellectual disability field. The panel members included social workers, psychiatric nurses, general nurses, occupational therapists, and physiotherapists. The review focused on the accuracy of translation, the comprehensibility and clarity of the instrument, and translation alternatives for items that were distorted from the original version. Further improvement of the instrument was performed, including the addition, deletion, or revision of words that did not appear in the original instrument and adjustment of grammar and syntax. The Chinese version of the instrument was subsequently ready for testing.

\section{Chinese interRAI ID}

The Chinese interRAI ID contains 273 items designed to assess the status of individuals with all levels of ID across all areas of life. Because the instrument intends to screen for a broad range of needs or problems-including functional status, cognition, psychosocial well-being and social supports, lifestyle, substance use, oral and nutritional status, health conditions, mood and behavior, mediations, environmental assessment, communication and vision, service utilization and interventions, education, employment, and recreation-only the minimum number of items needed to identify potential problem areas were included. Several scales, such as the ADL Hierarchy Scale, IADL Involvement Scale, DRS, CPS, and $A B S$, were embedded in the instrument.

\section{Cognitive Performance Scale}

The CPS is designed to measure cognitive status. It is a predictive algorithm based on a classification tree that describes general cognitive status. It includes 4 items-short-term memory, decision-making, expression, and self-performance in eating-combined in a hierarchical 7-category rating scale (refer to http://www.interrai.org/index.php?id=106 for more details). The overall scale score ranges from intact (0) to severely impaired (6). The CPS explained variation of .75 or slightly higher with the combined Mini-Mental State Examination and Test for Severe Impairment scores among residents of long-term care facilities, and was demonstrated to have a high correlation with the cognitive section of the Dementia Questionnaire for Persons with Mental Retardation $(r=.60, p<.0001)$ among community-dwelling adults with ID $[5,8]$.

\section{Depression Rating Scale}

DRS is an observer-rated scale that assesses depressive symptoms based on the presence of 7 indicators during the previous 3 days (made negative statement; persistent anger with self or others; expressions of what appear to be unrealistic fears; repetitive health complaints; repetitive anxious complaints/concerns; sad, pained, or worried facial expressions; and crying or tearfulness) [4]. The 7 items are rated on a 3 point-Likert scale based on observed frequency $(0=$ not present; 1 = present on 1 or 2 days; $2=$ present on all 3 days). Scale score ranges between 0 and 14. The internal consistency of DRS was found to be good $(\alpha=.78)$ and the scale was associated significantly with the depression subscale of the Reiss Screen for Maladaptive Behavior among persons with ID $(r=.65, p<.0001)$ [7].

\section{Aggressive Behavior Scale}

The ABS was employed to assess the aggressive behavior of participants. It indicates the level of aggression exhibited during the previous 3 days and is based on four items: verbal abuse, physical abuse, socially inappropriate disruptive behavior, and resistance to care [8]. Scale scores ranges between 0 and 12, with higher scores indicating higher levels of aggression. Previous research has shown that the ABS achieved satisfactory internal consistency $(\alpha=.74)$ and was significantly correlated with the aggression subscale of the Reiss Screen among persons with ID ( $r$ $=.60, p<.0001)[8]$.

\section{Activities of Daily Living Hierarchy Scale}

The ADL Hierarchy Scale is a hierarchical 7category rating scale and was used to measure participants' ADL functioning. Its scoring is based on a predictive classification tree that classifies ADL status 
into a 7 categories ranging from 0 (independent) to 6 (total dependence) using 4 items in the ADL content area [6]; namely, personal hygiene, toileting, locomotion, and eating (refer to http://www.interrai.org/ index.php?id=106 for more details). In previous research, the ADL Hierarchy Scale correlated significantly with the practical skills subscale of the Dementia Questionnaire for Persons with Mental Retardation $(r=.65, p<.0001)$ among persons with ID [7].

\section{IADL Involvement Scale}

The IADL Involvement Scale was used to measure participants' instrument activities of daily living. The scale is based on the summation of 7 IADL items: meal preparation, ordinary housework, managing finance, managing medications, phone use, shopping, and transportation. Each item is rated on a 6-point Likert scale ranging from 0 (independent) to 6 (total dependence). Total scores range from 0 to 42, with higher scores indicating higher capacity.

\section{Criterion Measures for interRAI ID}

\section{Dementia Questionnaire for People with Learning Disabilities (DLD)}

The DLD was used during the validation of the original version of interRAI ID and was selected to validate the CPS. The DLD was developed as a screening instrument to detect dementia in adults with ID and was widely used in research and clinical practice throughout Europe and the United Kingdom. It is a 50 -item, informant-based instrument; each item is scored on a 3-point Likert-type scale [11]. Eight summated subscales (short-term memory, long-term memory, spatial and temporal orientation, speech, practical skills, mood, activity and interest, and behavioral disturbance) and 2 overall scores are generated: the sum of cognitive scores (SCS) and the sum of social scores. The SCS describes cognitive functioning at a single point in time. A sensitivity score between .73 and 1 and a specificity score between .53 and .97 have been reported for the DLD [12]. In this study, only SCS was used.

\section{Chinese version of Aberrant Behavior Checklist (CABC)}

The CABC is a validated scale that is designed to assess the challenging behavior of people with moderate to severe mental disabilities in Hong Kong and was chosen to validate the ABS [13]. It is a behavioral observation rating scale and consists of 58 items rated in a 4-point Likert scale (ranging from not a problem to severe problem). The items are organized into 5 factor-analytic domains that explained $56.7 \%$ of variance: (1) lethargy and social withdrawal; (2) irritability, agitation, and crying; (3) hyperactivity; (4) stereotypic and self-injurious behavior; and (5) inappropriate speech. Satisfactory test-retest and interrater reliability were reported [13].

\section{Barthel Index (BI)}

The $\mathrm{BI}$ is a gold standard of ADL assessment [14] and was selected to validate the ADL Hierarchy Scale. It consists of 10 items: feeding, bathing, grooming, dressing, bowel control, bladder control, toilet transfers, chair and bed transfers, ambulation, and stair climbing. The total score of the $\mathrm{BI}$ ranges from 0 to 100 . Granger, Albrecht, and Hamilton [15] reported a testretest reliability of .89 . Construct validity was supported by factor analysis and yielded a single domain. The Cronbach's alpha for the Barthel Index was .95. The translated Chinese version had a test-retest reliability of $\mathrm{K}=.63$ to $1(p<.001)$, an interrater reliability of .63 to $.85(p<.001)$, and an internal consistency of $\alpha=.92$ $(p<.001)[16]$

\section{Modified Lawton Instrumental Activities of Daily Living Scale (ML-IADLS)}

The ML-IADLS is a commonly used assessment of IADL functioning and was chosen to validate the IADL Involvement Scale. It was developed to measure IADL performance and consists of 8 tasks (use of telephone, mode of transportation, shopping, responsibility for own medication, laundry, food preparation, housekeeping, and ability to handle finances). Each item is rated on a 5-point Likert scale: independent (7), modified independent (6), supervised (5); assisted (3); and dependent (1). It was demonstrated to have good psychometric properties with high sensitivity [17]. The validated Hong Kong Chinese version has a content validity agreement of $73 \%$ on representativeness and a $78 \%$ to $93 \%$ agreement on cultural relevancy. The intraclass correlation coefficient for interrater reliability was .99 and the test-retest reliability was .90 . It has a Cronbach's alpha of .86, showing good internal consistency.

\section{Psychiatric Assessment Schedule for the Adults with Developmental Disability Checklist (PAS-ADD Checklist)}

The PAS-ADD Checklist is a well-validated instrument designed to assess the psychopathology of people with mental disabilities and was selected to 
validate the DRS [18]. It is an informant-based screening tool composed of 25 items rated on a 4-point scale related to psychiatric symptoms observed during the previous 4 weeks. Eight factor subscales (e.g., depression, phobic anxiety, psychosis, etc.) were derived from factor analysis that explained $65.3 \%$ of variance [19]. The measure has adequate reliability and validity and established normative data with this population [20].

\section{Data Analysis}

Descriptive statistics were used to summarize the demographic information of study participants. The reliability of the scales was evaluated in two ways. Firstly, the internal consistency of the DRS, ABS, and IADL Involvement Scale was assessed using Cronbach's alpha. Secondly, the test-retest reliability of the scales was examined by calculating intraclass correlation coefficients for the DRS, ABS, and IADL Involvement Scale and kappa coefficients for the ADL Hierarchy Scale and CPS. The kappa coefficients were computed for these scales due to the nature of scale measurement; these scale scores are formulated based on a predictive classification tree instead of summing of item scores. The concurrent validity of interRAI ID measures with criterion measures were evaluated by using Pearson correlations and Spearman's correlations. All analyses were performed using SPSS version 19.

\section{RESULTS}

A sample of 100 randomly selected study participants was assessed with the interRAI ID and criterion measures; only 27 participants were reassessed with the interRAI ID after 3-4 weeks because 3 participants were not reassessed due to hospitalization. Table $\mathbf{1}$ presents the baseline characteristics of the participants, including gender (65\% male), age $(M=36.1, S D=11.1)$, and moderate or severe intellectual disability $(80 \%)$. Comorbidity of psychiatric or medical problems included epilepsy or seizure disorder $(22 \%)$, autism or autistic spectrum disorder (11\%), Down syndrome $(7 \%)$, or a combination. The majority resided at a hostel for people with severe mental disabilities $(60 \%)$.

Table 2 presents the descriptive statistics of interRAI ID scales. A majority of participants were categorized by the CPS as having moderate to severe cognitive impairment (72\%). Seventy-eight percent of study participants were independent or required
Table 1: Characteristics of Participants $(N=100)$

\begin{tabular}{|c|c|}
\hline Variable & $n^{\mathrm{a}}$ \\
\hline \multicolumn{2}{|l|}{ Gender } \\
\hline Male & 65 \\
\hline Female & 35 \\
\hline \multicolumn{2}{|l|}{ Age } \\
\hline$\leq 25$ & 20 \\
\hline $26-35$ & 31 \\
\hline $36-45$ & 27 \\
\hline $46-55$ & 18 \\
\hline $56-65$ & 3 \\
\hline $66-75$ & 1 \\
\hline \multicolumn{2}{|l|}{ Intellectual disability severity } \\
\hline Borderline & 1 \\
\hline Mild & 19 \\
\hline Moderate & 65 \\
\hline Severe & 15 \\
\hline \multicolumn{2}{|l|}{ Other psychiatric diagnosis } \\
\hline Autism or autism spectrum disorder & 11 \\
\hline Down syndrome & 7 \\
\hline Schizophrenia & 4 \\
\hline \multicolumn{2}{|l|}{ Other medical problem } \\
\hline Epilepsy or seizure disorder & 22 \\
\hline Diabetes mellitus & 4 \\
\hline Asthma & 3 \\
\hline Cerebral palsy & 2 \\
\hline Hypothyroidism & 2 \\
\hline Traumatic brain injury & 2 \\
\hline \multicolumn{2}{|l|}{ Type of residential service } \\
\hline Moderate disability hostel & 40 \\
\hline Severe disability hostel & 60 \\
\hline \multicolumn{2}{|l|}{ Length of residential stay } \\
\hline $0-3$ years & 80 \\
\hline More than 3 years & 20 \\
\hline
\end{tabular}

${ }^{a}$ Figures also reflect percentages because sample size was 100 .

supervision in terms of ADL. The average mean and standard deviation of other interRAI ID scales were: DRS $(M=2.4, S D=1.8)$; IADL Involvement Scale $(M=$ $15.0, S D=4.3)$; and $\mathrm{ABS}(M=1.7, S D=1.9)$.

\section{Internal Consistency and Test-Retest Reliability}

The internal consistency reliability of the DRS, ABS, and IADL Involvement Scale was computed: DRS = 
Table 2: Descriptive Statistics of interRAI ID Scale Scores

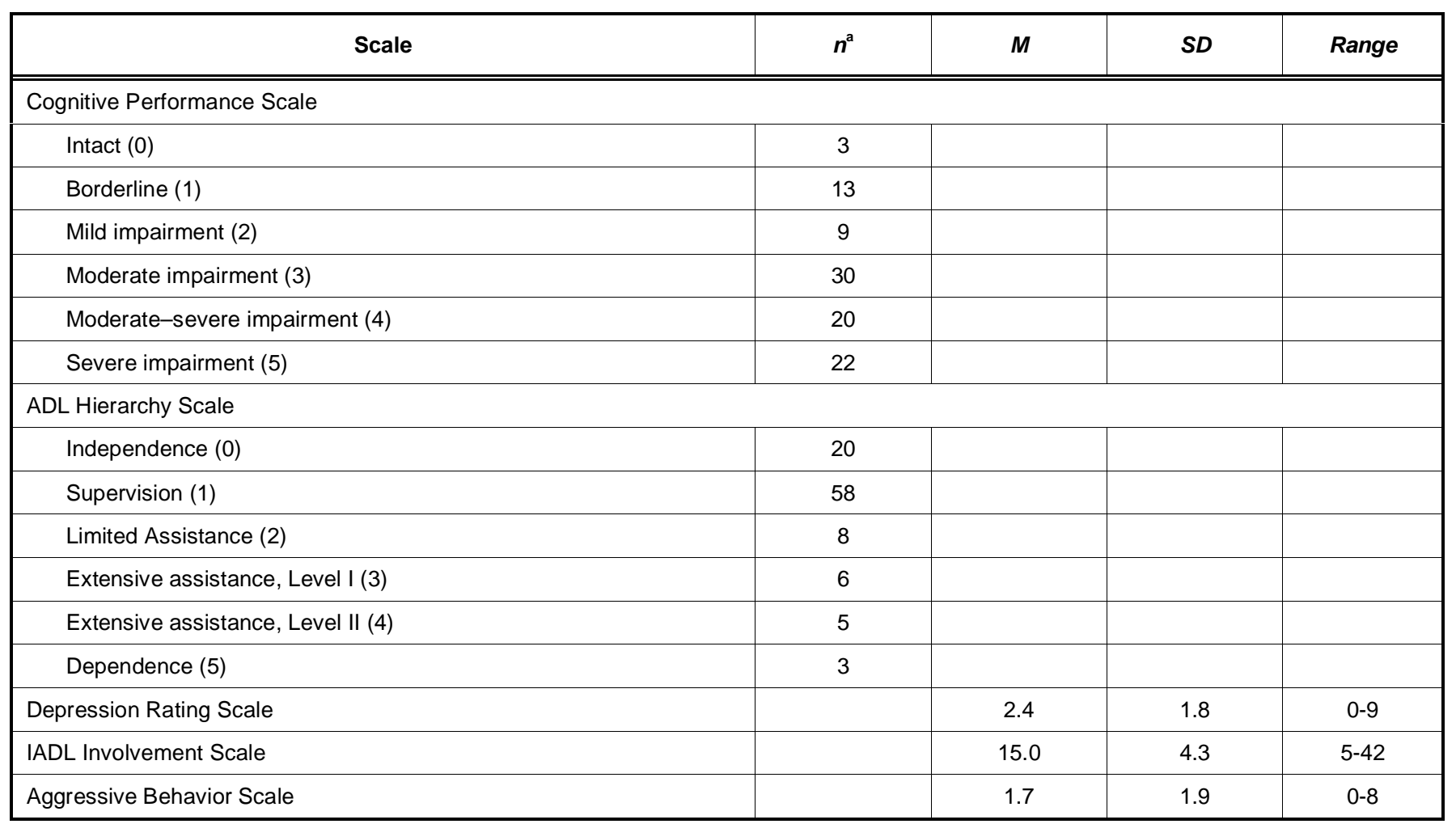

Note. $\mathrm{ADL}=$ activities of daily living, IADL = instrumental activities of daily living

${ }^{a}$ Figures also reflect percentages because sample size was 100 .

$.62 ; \mathrm{ABS}=66 ;$ and IADL Involvement Scale $=.87$. The coefficients were comparable to coefficients in previous research. With respect to stability of scale scores over time, the intraclass coefficients for the IADL Involvement Scale, ABS, and DRS and kappa coefficients for the CPS and ADL Hierarchy Scale were as follows: $\mathrm{DRS}=.96(p<.001)$; $\mathrm{ABS}=.99(p<.001)$; IADL Involvement Scale $=.99(p<.001) ;$ CPS $=.81(p$ $<.001)$; and ADL Hierarchy Scale $=.68(p<.001)$. These results show good stability of scale scores over time, suggesting that these scales measure relatively stable characteristics. Table $\mathbf{3}$ presents the internal consistency and test-retest reliability of the interRAI ID scales.

\section{Concurrent and Discriminant Validity}

Comparison of the strength of correlations between the interRAI ID scales and criterion measures were used to assess concurrent and discriminant validity (Table 4). There was a strong negative association between the ADL Hierarchy Scale and the BI $(r=-.66$, $p<.001)$ and between the IADL Involvement Scale and the ML-IADLS $(r=-.84, p<.001)$. Except for the correlation coefficient between the DRS and the

Table 3: Internal Consistency and Test-Retest Reliability of the interRAI Scales

\begin{tabular}{|c|c|c|c|c|}
\hline & \multicolumn{2}{|c|}{ Internal Consistency } & \multicolumn{2}{|c|}{ Test-Retest Reliability ${ }^{a}$} \\
\hline & Original $\alpha$ & Hong Kong a & Intraclass Correlation & K \\
\hline Depression Rating Scale & .78 & .66 & .96 & -- \\
\hline Aggressive Behavior Scale & .74 & .66 & .99 & -- \\
\hline IADL Involvement Scale & -- & .87 & .99 & -- \\
\hline Cognitive Performance Scale & $\mathrm{N} / \mathrm{A}$ & $\mathrm{N} / \mathrm{A}$ & -- & .81 \\
\hline ADL Hierarchy Scale & $\mathrm{N} / \mathrm{A}$ & $\mathrm{N} / \mathrm{A}$ & -- & .68 \\
\hline
\end{tabular}

Note. $\mathrm{IADL}=$ instrumental activities of daily living, $A D L=$ activities of daily living.

${ }^{a}$ Mean interval between test and retest was 27 days.

N/A: Scale scores of CPD and ADL Hierarchy Scale are formulated based on a predictive classification tree instead of summing of item scores. It is not appropriate to compute Cronbach's alpha of these scales. Kappa statistic was used to evaluate the test-retest reliability of these scales as it measures the agreement between the scale scores at 2 different periods. 
Table 4: Concurrent and Discriminant Validity of the interRAI ID Scales

\begin{tabular}{|l|c|c|}
\hline \multicolumn{1}{|c|}{ interRAI Scale } & Validation Scale & $\mathbf{Y}$ \\
\hline \hline Cognitive Performance Scale $^{\mathrm{a}}$ & DLD Sum of Cognitive Score & $.66^{* * *}$ \\
& PAS-ADD Checklist & .03 \\
\hline Aggressive Behavior Scale & CABC Irritability & $.81^{\text {*** }}$ \\
\hline Depression Rating Scale & CABC Inappropriate Speech & .11 \\
& PAS-ADD Checklist & $.28^{\text {b* }}$ \\
\hline ADL Hierarchy Scale & DLD Sum of Cognitive Score & $.26^{* *}$ \\
\hline IADL Involvement Scale & Barthel Index & $-.66^{* * *}$ \\
& ML-IADLS & -.17 \\
\hline
\end{tabular}

Note. Spearman correlation coefficients are reported for the CPS and the ADL Hierarchy Scale. Pearson correlation coefficients are reported for the ABS and the IADL Involvement Scale. $A D L=$ activities of daily living, IADL = instrumental activities of daily living, $D L D=$ Dementia Questionnaire for People with Learning Disabilities, PAS-ADD = Psychiatric Assessment Schedule for the Adults with Developmental Disability Checklist, CABC = Chinese version of Aberrant Behavior Checklist, ML-IADLS = Modified Lawton Instrumental Activities of Daily Living Scale.

${ }^{a}$ Sample size was 97 instead of 100 as missing data in items of CPS was found in 3 study participants.

${ }^{\mathrm{b}}$ Refers to the depression subscale of the PAS-ADD Checklist.

depression subscale of the PAS-ADD Checklist $(r=$ $.28, p<.01$ ), a moderate-to-strong positive relationship was found between scales and criterion measures, including the $A B S$ and the irritability subscale of the ABC $(r=.81, p<.001)$ and the CPS and the SCS of the DLD $(r=.66, p<.001)$.

On the other hand, no significant association was found between the CPS and the depression subscale of the PAS-ADD Checklist $(\rho=.03, p=.80)$, between the ABS and the inappropriate subscale of the CABC $(r$ $=.11, p=.26)$, and between the ADL Hierarchy Scale and the ML-IADLS $(\rho=-.17, p=.10)$. However, a significant association was found between the DRS and the SCS of the DLD $(r=.26, p=.01)$ and the IADL Involvement Scale and the $\mathrm{BI}(r=-.44, p=.000)$. All interRAI ID scale scores were independent of age and gender of study participants.

\section{DISCUSSION}

This preliminary study attempted to examine the reliability (internal and test-retest) and validity (concurrent and discriminant) of 5 scales of interRAI ID. Study results indicated that the Chinese interRAI ID has good reliability and validity, supporting its use to assess the needs and strengths of people with learning disabilities in the Hong Kong context.

The results of reliability analysis revealed that the internal consistency of the 3 scales of the Chinese interRAI ID ranged from .66 to .87 , which suggests good internal consistency. Additionally, the coefficients are comparable to those found for the original version in previous research $[4,8]$. Also, test-retest reliability estimates for the 5 scales of the Chinese interRAI ID ranged from .68 to .99 . These scales are considered to have good test-retest reliability.

In terms of concurrent validity, all of the scales of the Chinese interRAI ID correlated moderately well at statistically significant levels with corresponding criterion measures. Our concurrent validity results for the CPS and ABS were consistent with the original validation study of the interRAI ID [7, 8]. A small but significant correlation between the DRS and both the depression subscale of the PAS-ADD Checklist and the DLD's SCS suggests that the construct of depression may be not fully captured by the DRS among people with moderate and severe ID. The results were not consistent with the validation of the original version of the instrument [7]. This may be explained by the fact that our study included participants who predominately had moderate to severe ID, whereas the original validation study included participants with a wide range of intellectual impairments. Some have questioned the usefulness of current classification systems (i.e., International Classification of Diseases 10th edition and the Diagnostic and Statistical Manual of Mental Disorders 4th edition) in detecting depressive symptoms among people with moderate, severe, and profound levels of ID [21]. Given the prevalence rate of affective disorders among people with ID-as high as $3.8 \%$ in a recent epidemiological study [22]-further studies recruiting individuals with a wide range of 
intellectual impairments will be needed to examine the validity of DRS in screening for depressive symptoms in this population. Moreover, clinical features are often poorly defined among those with intellectual impairments, rendering diagnosis difficult [23].

Concerning the discriminant validity of the interRAI scales, we found a moderate association between the IADL Involvement Scale and the $\mathrm{BI}$, suggesting an overlap of constructs between the two measures. Comparing the strength of the correlation coefficient between the IADL Involvement Scale and both the BI and the ML-IADLS provides strong support for the IADL Involvement Scale as a measure of IADL constructs. In fact, a moderate association between the ADL and IADL functioning has been commonly noted in various client groups, such as cancer patients and frail older adults [24, 25].

\section{CONCLUSION AND LIMITATIONS}

Our sample was recruited from an agency in Hong Kong that provides residential care services only for individuals with moderate to severe ID. Generalization of our results to people with ID in other settings in Hong Kong is limited. Additionally, the sample size of the study prevented us from employing advanced statistical methods to examine the construct validity of the scales. Future research should recruit participants from various settings with a larger sample size to reveal the construct validity of interRAI scales and its clinical utility in this population.

In conclusion, the Chinese interRAI ID can be regarded as a useful tool for assessment of people with ID residing in residential settings by virtue of its good internal consistency, test-retest reliability, and concurrent and discriminant validity with criterion measures. However, the depression subscale of the tool needs further validation. In general, the interRAI ID is a valid and reliable instrument that can be recommended for use in the clinical setting.

\section{ACKNOWLEDGEMENTS}

The essential contribution made to this research by the Hong Kong Sheng Keung Hui Welfare Council and the participating clinicians and service users of the Providence Garden for Rehab is very gratefully acknowledged. The specific assistance provided by Dr. Vivian Lou in obtaining ethical approval is also gratefully acknowledged. At the time of the study, the first author worked at the Chinese University of Hong Kong.

\section{CONFLICT OF INTEREST}

The authors declare no conflicts of interest.

\section{REFERENCES}

[1] Nihira K, Leland H, Lambert N. AAMR Adaptive Behavior Scale: residential and community: examiner's manual. $2^{\text {nd }}$ ed. Austin, TX: Pro-Ed 1993.

[2] Sparrow SS, Balla DA, Cicchetti DV. Vineland Adaptive Behavior Scales: interview edition (survey form). Circle Pines, MN: American Guidance Services 1984

[3] Hirdes JP, Martin L, Curtin-Telegdi N, et al. interRA Intellectual Disability (ID): primer for use of the interRAI ID Assessment Form and Clinical Assessment Protocols (CAPs). Washington, DC: interRAI 2007.

[4] Burrows AB, Morris JN, Simon SE, Hirdes JP, Phillips C Development of a Minimum Data Set-based depression rating scale for use in nursing homes. Age Ageing 2000; 29: 165-72.

http://dx.doi.org/10.1093/ageing/29.2.165

[5] Morris JN, Fries BE, Mehr DR, et al. MDS Cognitive Performance Scale. J Gerontol Med Sci 1994; 49: M174-82.

[6] Morris JN, Fries BE, Morris SA. Scaling ADLs within the MDS. J Gerontol Med Sci 1999; 54A: M546-53.

[7] Martin L, Hirdes JP, Fries BE, Smith TF. Development and psychometric properties of an assessment for persons with intellectual disability: the interRAI ID. J Pol Pract Intellect Disabil 2007; 4: 23-9.

http://dx.doi.org/10.1111/j.1741-1130.2006.00094.x

[8] Perlman CM, Hirdes JP. The Aggressive Behavior Scale: a new scale to measure aggression based on the Minimum Data Set. J Am Geriatr Soc 2008; 56: 2298-303. http://dx.doi.org/10.1111/j.1532-5415.2008.02048.x

[9] Anderson RL, Buckwalter KC, Buchanan RJ, Maas ML, Imhof SL. Validity and reliability of the Minimum Data Set Depression Rating Scale (MDSDRS) for older adults in nursing homes. Age Ageing 2003; 32: 435-8. http://dx.doi.org/10.1093/ageing/32.4.435

[10] Cohen J. Statistical power analysis for the behavioral sciences. 2nd ed. Hillsdale, NJ: Lawrence Erlbaum 1988.

[11] Evenhuis HM, Kengen MMF, Eurlings HAL. Dementia Questionnaire for People with Learning Disabilities (DLD) (UK adaptation). London: Harcourt 2007.

[12] Evenhuis HM. Evaluation of a screening instrument for dementia in ageing mentally retarded persons. J Intell Disabil Res 1992; 36: 337-47. http://dx.doi.org/10.1111/j.1365-2788.1992.tb00532.x

[13] Lee WKS. A study of the reliability and validity of the Chinese version Aberrant Behavior Checklist. Master's thesis. Hong Kong: Hong Kong Polytechnic University 2000.

[14] Mahoney FI, Barthel DW. Functional evaluation: the Barthe Index. Md State Med J 1965; 14: 61-5.

[15] Granger CV, Albrecht GL, Hamilton BB. Outcome of comprehensive medical rehabilitation: measurement by PULSES profile and the Barthel Index. Arch Phys Med Rehabil 1979; 60: 145-54

[16] Leung SOC, Chan CCH, Shah S. Development of a Chinese version of the Modified Barthel Index: validity and reliability. Clin Rehabil 2007; 21: 912-22.

http://dx.doi.org/10.1177/0269215507077286

[17] Edwards MM. The reliability and validity of self-report activities of daily living scales. Can J Occup Ther 1990; 57: 273-8.

http://dx.doi.org/10.1177/000841749005700507 
[18] Moss S, Prosser H, Costello H, Simpson N, Patel P. PASADD Checklist. Manchester, England: Hester Adrian Research Centre, University of Manchester 1996.

[19] Moss $\mathrm{S}$, Prosser $\mathrm{H}$, Costello $\mathrm{H}$, et al. Reliability and validity of the PAS-ADD Checklist for detecting psychiatric disorders in adults with intellectual disability. J Intell Disabil Res 1998; 42: 173-83.

http://dx.doi.org/10.1046/j.1365-2788.1998.00116.x

[20] Taylor JL, Hatton C, Dixon L, Douglas C. Screening for psychiatric symptoms: PAS-ADD Checklist norms for adults with intellectual disabilities. J Intell Disabil Res 2004; 48: 3741.

http://dx.doi.org/10.1111/j.1365-2788.2004.00585.x

[21] Perez-Achiaga N, Nelson S, Hassiotis A. Instruments for the detection of depressive symptoms in people with intellectual disabilities: a systematic review. J Intellect Disabil 2009; 13: 55-76. http://dx.doi.org/10.1177/1744629509104487
[22] Cooper SA, Smiley E, Morrison J, Williamson A, Allen L. An epidemiological investigation of affective disorders with a population-based cohort of 1023 adults with intellectual disabilities. Psychol Med 2007; 37: 873-82.

http://dx.doi.org/10.1017/S0033291707009968

[23] Masi G, Pfanner P, Marcheschi M. Depression in adolescents with mental retardation: a clinical study. Br J Dev Disabil 1998; 44: 112-8. http://dx.doi.org/10.1179/096979598799156065

[24] Chan CLF, Chui EWT. Association between cultural factors and the caregiving burden for Chinese spousal caregivers of frail elderly in Hong Kong. Aging Ment Health 2011; 15: 5009. http://dx.doi.org/10.1080/13607863.2010.536139

[25] Peel C, Baker PS, Roth DL, Brown CJ, Bodner EV, Allman RM. Assessing mobility in older adults: the UAB Study of Aging Life-Space Assessment. Phys Ther 2005; 85: 1008-19. 